\title{
Evaluation of some Faba Bean Cultivars Yield under Chemical Control to Common Insect Pests and Fungal Diseases under El-Beheira Governorate Environmental Conditions Waly, F. E. ' ; E. A. Rashwan ${ }^{2}$; Gehad M. Abel-Whab ${ }^{3}$; I. F. Khafagy ${ }^{4}$ and Hanim M. Arafa ${ }^{5}$ \\ ${ }^{1}$ Food Legume Research Section, Field Crops Research Institute, A.R.C. \\ ${ }^{2}$ Agronomy Department, Fac. of Agri. Tanta Univ. \\ ${ }^{3}$ Plant Diseases Institute, A.R.C, Dokki, Giza. \\ ${ }^{4}$ Plant Protection Institute, A.R.C, Dokki, Giza. \\ ${ }^{5}$ Central Lab for Pesticides, A.R.C, Dokki, Giza. \\ Corresponding author e-mail: emad.rashwan@agr.tanta.edu.eg
}

\section{ABSTRACT}

In order to investigate six faba bean cultivars Nubaria 1, Sakha 1, Giza 716, Giza 843, Misr 1 and Giza 40 under foliar spraying with Actara insecticide (thiamethoxam), Sumi-8 fungicide (diniconazole) and thiamethoxam + diniconazole compared to control for yield and its components, some biochemicals, leaf miner (Liromiza congesta) numbers, aphid (Aphis gossypii) numbers, chocolate spot disease severity caused by Botrytis fabae Sard. and pesticides residue in green pods. The results showed that, Giza $716 \mathrm{cv}$. surpassed other cultivars in plant height, number of branches/plant, number of pods/plant, seed yield/plant and seed yield/plot in both seasons. Giza $40 \mathrm{cv}$. showed the highest values for chlorophyll a, chlorophyll $\mathrm{b}$, total carbohydrate $\%$, total protein $\%$, peroxidase activity and polyphenoloxidase activity in both seasons respectively. Faba bean plants under the Actara insecticides (thiamethoxam) recorded the highest mean values for plant height, number of branches/plant, number of pods/plant, 100-seed weight, seed yield/plant, seed yield/plot and lowest aphid and leaf miner (Liromiza congesta) numbers in both seasons. While, the control treatment gave the highest mean values for chlorophyll a, total protein, peroxidase activity and polyphenol-oxidase activity in both seasons respectively. Diniconazole and thiamethoxam + diniconazole treatments showed the lowest disease severity of chocolate spot caused by Botrytis fabae. The lowest numbers of leaf miner (Liromiza congesta) in both seasons were counted in Misr $1 \mathrm{cv}$. followed by Nubaria 1 then Giza 40. In the same way, the lowest numbers of aphid were collected from Giza 40 followed by Misr 1 then Nubaria 1 in the two seasons. Also, Nubaria 1 $\mathrm{cv}$. was the most resistant one to chocolate spot where it scored the lowest disease severity among all cultivars in both seasons followed by Misr $1 \mathrm{cv}$. in both seasons. With regard to residues analysis, the half-life value of thiamethoxam was 3.02, 3.59, and 2.57 days of the application with degradation rate of $0.138,0.051$ and 0.269 for pods, peels and seeds, respectively.

Keywords: Faba bean, Vicia faba L., Cultivars, Chlorophyll a and b, Aphid (Aphis gossypii), Leaf miner (Liromiza congesta), chocolate spot.

\section{INTRODUCTION}

Faba bean (Vicia faba L.) is one of the most important food legumes due to its high nutritive value both in terms of energy and protein content (24-30\%) and is an excellent nitrogen fixer (Abebe et al., 2014). The world total production of dry seeds were 4.82 million tons produced from 2.46 million hectares while, the Egyptian total production of dry seed were 112.87 thousand tons produced from 32.53 thousand hectares (FAOSTAT. 2017). In Egypt, faba bean is an important food pulse crop (Hendawey and Younes, 2013). It is pivotal to evaluate faba bean' cultivars especially the recent improved ones, the report of ICARDA (2008) revealed that shifting fromn traditional cultivars to improved ones could enhance faba bean yield about 18\% in Egypt, $8 \%$ in Sudan and 42\% in Ethiopia. Variations among faba bean cultivars were noted in many previous studies, such as: (Zebire and Tadesse 2018) when they evaluated ten improved faba bean cultivars and two local controls, they found a significant variations among the faba bean cultivars for plant height, number of dry pods plant ${ }^{-1}$, yield of dry seed and 1000 seed weight traits. Furthermore, (Mitiku and Wolde 2015) evaluated eight improved faba bean cultivars during the main cropping season and they reported significant variations among these cultivars for effective tillers plant ${ }^{-1}$, plant height, number of pods plant ${ }^{-1}$, number of seeds pod $^{-1}$, days to $90 \%$ physiological maturity, 100 grain weight, and grain yield. In most cases, biotic and abiotic factors are the main reason of the low productivity of faba bean (Agegnehu et al., 2006). This crop infested by numerous of pests such as Aphis craccivora and Liriomyza trifolii (Abou-Elhagag and Salman, 2001). Aphis craccivora causes direct damage by feeding, which may induce plant deformation and indirect damage caused either by honeydew or by transmission of viruses (ElDefrawi et al., 2000), also larvae of Liriomyza trifolii eat the mesophyll of leaf least leaving long winding tunnels inside the leaflets and form numerous mines that reduce the capacity of plants photosynthetic activity (Ali and Morsy (1983), Bueno (2007) and Baliad and Tengkano (2010). Diseases are among the important biotic constrains that limit the production of faba bean crop. Chocolate spot (Botrytis fabae Sard.) and rust (Uromyces fabae Pers. Schart) are the economically important diseases that damage the foliage, limiting photosynthetic activity and reduce faba bean production (Torres et al., 2006).

Faba bean genotypes had a different tolerance to insects and fungi diseases. The resistant genotypes may produce high yield under low and moderate infection but in the high infections the yield production will decreased in the resistance and susceptible genotypes together. In the critical infections the chemical control will be useful in reduced yield loss caused by pestes.

The aim of this study were to evaluate seed yield of six Egyptian faba bean cultivars under chemical control to aphid, leaf miner (Liromiza congesta) and chocolate spot disease using some growth, yield and some chemical components of faba bean plants under field conditions.

\section{MATERIALS AND METHODS}

Two field experiments were conducted at experimental farm of Itay El-Baroud Agricultural Research Station during the two successive seasons of 2016/17 and 2017/18. The six faba bean cultivars Nubaria 1, Sakha 1, 
Giza 716, Giza 843, Misr 1 and Giza 40 were evaluated for seed yield and other important agronomic traits under foliar spraying with Actara insecticide (thiamethoxam), Sumi-8 fungicide (diniconazole) and thiamethoxam + diniconazole compared to control. The treatments were arranged in a strip plot design with three replications, whereas cultivars and chemical control treatments were randomly assigned separately and independently in the vertical and horizontal strips, respectively. The plot size was 5 ridges each ridge was three meters long and $70 \mathrm{~cm}$ apart. Seeds were planted in two sides of the ridge at 15 $\mathrm{cm}$ hill spacing with one seed hill ${ }^{-1}$. Faba bean plants were sprayed with the Actara insecticide (thiamethoxam) three times, at ages of 40, 60 and 75 using the recommended dosage feddan ${ }^{-1}$ for each pesticide, while control was sprayed with tap water only. All necessary agronomic practices were done as required.

\section{Data collection}

1- Crop data:

Ten plants from each plot were randomized chosen to measure the average of plant height $(\mathrm{cm})$, numbers of branches/plant, number of pods/plant, 100-seed weight (g) and seed yield/plant $(\mathrm{g})$ and seed yield/feddan $(\mathrm{kg})$ which estimated as the total seed yield for each sub plot, then converted into $\mathrm{kg} /$ feddan.

2- Determination of leaf chlorophyll:

Chlorophyll $\mathrm{a}$ and $\mathrm{b}$ were determined as $\mathrm{mg} \mathrm{g}^{-1}$ fresh weight quantitatively using $\mathrm{N}$, Ndimethyliformamide (DMF) as described by (Moran and Porath, 1980). Leaves were selected from different positions on the faba bean stem after 90 days from sowing and immersed directly in pure DMF $\left(2-5 \% \mathrm{w} \mathrm{v}^{-1}\right)$ then kept in the dark for $1-2$ days at $4 \mathrm{C}^{\circ}$. The extracts were centrifuged for $15 \mathrm{~min}$ at $3000 \mathrm{rpm}$, then the supernatant diluted to the appropriate volume. The extinction of the extracts was measured spectrophotometrically against a blank of pure DMF at $646.8,663.8$ and $750 \mathrm{~nm}$. The following equations were applied for determining the Chl. content of the leaf sample:

$$
\text { Chl a }=12.00\left(E_{663.8}-E_{750}\right)-3.11\left(E_{646.8}-E_{750}\right)
$$$$
\text { Chl b }=20.78\left(E_{646.8}-E_{750}\right)-4.88\left(E_{663.8}-E_{750}\right)
$$

3- Determination of total carbohydrate and protein in dried seeds:

Total carbohydrate was determined using phenol sulphuric method (Dubois et al., 1956). Total nitrogen percentage was determined by Modified Micro-Kjeldahl method as described by (AOAC 1995) and the percentage of protein was calculated by multiplying total $\mathrm{N}$ values by factor 6.25.

\section{4- Determination of Enzyme Activities:}

The sample of one $\mathrm{g}$ of leaves (after 90 days from sowing) was homogenized in $2 \mathrm{ml}$ of $0.1 \mathrm{M}$ sodium phosphate buffer (SPB) $\mathrm{pH} 6.5$ at $4^{\circ} \mathrm{C}$. The filtrate was centrifuged at $20,000 \mathrm{rpm}$ at $4^{\circ} \mathrm{C}$ for $15 \mathrm{~min}$., the supernatant served as an enzyme extract for enzyme assay of polyphenoloxidase and peroxidase.

1-Peroxidase activity: Peroxidase activity was assayed colorimetrically according to the method described by (Amako et al., 1994). The increase in optical density at $430 \mathrm{~nm}$ against blank was continuously recorded every minute. Peroxidase enzyme activity was expressed as change in absorbance per $\mathrm{min} / \mathrm{g}$ fresh leaves.
2- Polyphenol oxidase (PPO) activity: Polyphenol oxidase activity was estimated as described by Mayer and Harel (1979) with some modifications. The polyphenol oxidase activity was expressed as change in absorbance at $495 \mathrm{~nm}$ against blank per min $\mathrm{g}^{-1}$ fresh leaves.

5- Number of leaf miner (Liromiza congesta) and aphid (Aphis gossypii):

Monitoring of B. tabaci and Aphis gossypii, numbers was conducted throughout the from three after sowing until the end of the fruiting stage in the six faba bean cultivar plots. Sampling was carried out at weekly interval early in the morning before the insect pests adults tend to be more active, (Gameel et al., 1973). Number of whitefly and aphid were assessed by leaf random sampling. For sampling, 30 leaves representing top, middle and bottom canopy were picked from each of the six faba bean varsities were randomly selected per plot. Thus, in all 120 leaves replication ${ }^{-1}$ were observed at one time for each cultivar. Leaf samples were kept in separate paper bags properly labeled with plot number. The leaves were then brought to the laboratory on the same day where the adult insect pests were counted.

6- Determination of chocolate spot disease severity:

Severity due to natural infection was determined after 75, 90 and 105 days from sowing. Ten randomly pretagged faba bean plants in the three central rows, disease severity on leaves was rated using 1-9 rating scale (Bernier et al.,1993) according to the following formula:

Where:

Disease severity $\%=\Sigma(n \times v) / 9 N) \times 100$

(n) = Number of plants in each category;

(v) = Numerical values of symptoms category;

(N) = Total number of plants;

(9) = Maximum numerical value of symptom category.

Then efficacy percentage (E \%) of each compound in reducing disease, severity percentage of faba bean was assessed according to the equation adapted by Rewal and Jhooty (1985) as follow:

Where:

$$
\mathrm{E} \%=(\mathrm{C}-\mathrm{T} / \mathrm{C}) \times 100
$$

$\mathrm{C}=$ Disease severity $\%$ in control treatment;

$\mathrm{T}=$ Disease severity $\%$ in the treatment.

Statistical analysis: All data were subjected to the analyses of variance (ANOVA) for strip-plot design followed by compared means with LSD at level probability $5 \%$ according to Gomez and Gomez (1984).

\section{RESULTS AND DISCUSSION}

1- Effect of cultivars, pesticides and their interaction on plant height $(\mathrm{cm})$, number of branches/plant, number of pods/plant, 100-seed weight $(\mathrm{g})$, seed yield/plant (g) and seed yield/plot $(\mathrm{kg})$ in 2016/17 and $2017 / 18$ growing seasons:

\section{1- Cultivars effect:}

The presented results in Table (1) indicated that Sakha $1 \mathrm{cv}$. had excellent mean values for plant height in both seasons respectively with averages of 107.08 and 107.67 and number of pods/plant in the $2^{\text {nd }}$ season (12.58). The faba bean cultivars Giza 716 scored the excellent mean values for plant height $(111.67$ and $108.33 \mathrm{~cm})$, number of branches/plant (2.98 and 2.63), number of pods/plant (12.60 and 12.46), seed yield/plant (19.44 and 
$18.79 \mathrm{~g}$ ) and seed yield/fed. (1184 and $1136 \mathrm{~kg}$ ) followed by Giza 843 and Misr 1 in $1^{\text {st }}$ and $2^{\text {nd }}$ seasons respectively. On the other side, Giza $40 \mathrm{cv}$. seemed to be the worst among all genotypes where it showed the lowest mean values for yield and yield components traits and most growth traits in both seasons.

Our results are in disagree with those by Bakry et al. (2011) who reported that Nubaria 1 variety presented the first class in number of branches, pods and seeds weight per plant, 100 seed weight, seed and straw yields . Also, Abo-Khalil et al. (2015) who found that Nubaria 1 variety achieved the superiority on seed yield/ feddan, number of branches per plant, seeds weight and 100 - seed.

\section{2- Pesticides effect:}

The results in Table (1) showed that, faba bean plants under the Actara insecticides (thiamethoxam) give the highest mean values for plant height (106.39 and 108.44), number of branches/plant (2.34 and 2.44), number of pods/plant (12.13 and 12.02), 100-seed weight (84.29 and $84.73 \mathrm{~g}$ ), seed yield/plant (19.43 and $18.59 \mathrm{~g}$ ) and seed yield/fed. (1320 and $1284 \mathrm{~kg}$ ) in both seasons respectively. Where, spraying faba bean plants with thiamethoxam + diniconazole showed excclent mean values for plant height $(106.94 \mathrm{~cm})$ and number of branches $(2.44)$ in the $1^{\text {st }}$ season. While, spraying faba bean plants with the diniconazole exceeded all other treatment in 100-seed weight in the $2^{\text {nd }}$ season $(85.15 \mathrm{~g})$. Also, the data indicated that treated faba bean plants with thiamethoxam and diniconazole exceeded the control and thiamethoxam + diniconazole in all yield and yield component traits.

In this study pesticides may helped plants in reducing the loss of yield caused by the insect damage and this finding has been reported before by Cheema et al. (2009) which evaluated seven insecticides (thiamethoxam, triazophos, ethion, endosulfan, dimethoate, oxydemeton methyl and malathion) for their efficacy against whitefly, B. tabaci on urd bean, Vigna mungo (Linnaeus) during Autumn 2004-2006. They found that Thiamethoxam 25 WG, $100 \mathrm{~g} /$ ha gave the highest mean grain yield and net returns followed by endosulfan $35 \mathrm{EC}, 2.51 /$ ha and triazophos $40 \mathrm{EC}, 1.5 \mathrm{l} / \mathrm{ha}$. Teshome and Tagegn (2013) and El-Kholy, (2014) who found that spraying with fungicides gave the best results in reducing the disease incidence and severity, improved plant growth and finally increased grain yield.

\section{3- Cultivars $x$ pesticides effects:}

The results presented in Table (2) indicated that the six cultivars had different responses to the four treatments. The results showed that the interactions between Giza 716, Nubaria and Misr 1 with thiamethoxam, diniconazole and thiamethoxam + diniconazole had excellent mean values for plant height, number of branches/plant, number of pods, 100-seed weight, seed yield / plant and seed yield/plot in both seasons. The highest responses among these three cultivars were obtained from Giza 716 and Nubaria 1 with the thiamethoxam treatment in both seasons. On the other hand, the lowest response for yield and yield components taits in this study were obtained by Giza 40 with all treatments in both seasons.

2- Effect of cultivars, pesticides and their interaction on Chlorophyll a, Chlorophyll b, Total carbohydrate $(\%)$,Total protein $(\%)$, Peroxidase activity and polyphenoloxid activity in 2016/17 and 2017/18 growing seasons:

\section{1- Cultivars effect:}

The presented results in Table (3) indicated that, Sakha $1 \mathrm{cv}$. had high mean values for chlorophyll $\mathrm{b}$ content in both seasons respectively with averages of 0.53 and 0.54 , peroxidase activity in the $1^{\text {st }}$ season $(0.52)$ and polyphenoloxidase activity in both seasons with averages of 0.49 and 0.51 respectively. The faba bean cultivars Giza 843 scored high mean values for chlorophyll a $(0.78$ and $0.81)$, chlorophyll $\mathrm{b}(0.56$ and 0.57$)$, total carbohydrate $\%$ (51.67 and 53.22), peroxidase activity ( 0.55 and 0.57$)$ and polyphenoloxidase activity $(0.52$ and 0.54$)$ in $1^{\text {st }}$ and $2^{\text {nd }}$ seasons respectively. Giza $40 \mathrm{cv}$. showed the highest values for chlorophyll a $(0.80$ and 0.82$)$, chlorophyll $b$ (0.56 and 0.58$)$, total carbohydrate percentage ( 51.75 and 53.12), total protein percentage (24.21 and 24.85), peroxidase activity $(0.54$ and 0.56$)$ and polyphenoloxidase activity $(0.52$ and 0.53$)$ in $1^{\text {st }}$ and $2^{\text {nd }}$ seasons respectively.

On the other side, Giza $40 \mathrm{cv}$. Seemed to the worst among all genotypes where it showed the lowest mean values for yield and yield components traits and most growth traits in both seasons.

Our results in disagree with those by Bakry et al. (2011) who reported that Nubaria 1 variety presented the first class in carbohydrate percentage. Also, Abo-Khalil et al. (2015) found that the superiority went in harvest index and protein percentage to Sakha 1 variety.

\section{2- Pesticides effect:}

The obtained results in Table (3) showed that, in both seasons spraying faba bean plants with thiamethoxam, diniconazole and thiamethoxam + diniconazole led to increase pigments content and decreased total protein, peroxidase activity and polyphenol-oxidase activity compared to control. The control treatment gave the highest mean values for chlorophyll a (0.78 and 0.80$)$, total protein $(23.55$ and 24.24$)$, peroxidase activity $(0.68$ and $0.70)$ and polyphenol-oxidase activity (0.64 and 0.66$)$ in both seasons, respectively. Moreover, it exceeded the thiamethoxam and diniconazole treatments in total carbohydrate in the $2^{\text {nd }}$ season (52.36). In the same way, the thiamethoxam + diniconazole treatment showed the highest chlorophyll $\mathrm{b}$ contents $(0.57$ and 0.58$)$ and total carbohydrate (51.71 and 52.73\%) in both seasons respectively. On the other hand faba bean plants showed the lowest pigments content under control treatment, the lowest total carbohydrate percentage, total protein percentage under diniconazole treatment and the lowest peroxidase and poly-phenol oxidase activity under thiamethoxam + diniconazole compared to all other treatments in both seasons. 
Waly, F. E. et al.

Table 1. Means of plant height (cm), number of branches/plant, number of pods/plant, 100-seed weight (g), seed yield/plant (g) and seed yield/plot (kg) as affected by faba bean cultivars and pesticides in 2016/17 and 2017/18 growing seasons.

\begin{tabular}{|c|c|c|c|c|c|c|c|c|c|c|c|c|}
\hline \multirow{2}{*}{$\begin{array}{l}\text { Traits } \\
\text { Factors }\end{array}$} & \multicolumn{2}{|c|}{$\begin{array}{l}\text { Plant height } \\
\text { (cm) }\end{array}$} & \multicolumn{2}{|c|}{$\begin{array}{c}\text { No. of } \\
\text { branches / plant }\end{array}$} & \multicolumn{2}{|c|}{$\begin{array}{c}\text { No. of } \\
\text { Pods / plant }\end{array}$} & \multicolumn{2}{|c|}{$\begin{array}{l}\text { 100-seed } \\
\text { weight (g) }\end{array}$} & \multicolumn{2}{|c|}{$\begin{array}{l}\text { Seed yield/plant } \\
(\mathrm{g})\end{array}$} & \multicolumn{2}{|c|}{$\begin{array}{c}\text { Seed yield/feddan } \\
(\mathrm{kg})\end{array}$} \\
\hline & 2016/17 & $2017 / 18$ & $2016 / 17$ & $2017 / 18$ & $2016 / 17$ & $2017 / 18$ & $2016 / 17$ & 2017/18 & 2016/17 & $2017 / 18$ & $2016 / 17$ & $2017 / 18$ \\
\hline \multicolumn{13}{|c|}{$\begin{array}{ll} & \text { Cultivars }\end{array}$} \\
\hline Nubaria 1 & 107.08 & 107.67 & 2.40 & 2.27 & 11.66 & 12.58 & 82.68 & 83.66 & 16.07 & 15.41 & 1148 & 1096 \\
\hline Sakha 1 & 100.42 & 103.75 & 2.33 & 2.17 & 9.59 & 9.42 & 78.15 & 78.72 & 15.73 & 14.75 & 1072 & 1052 \\
\hline Giza 716 & 111.67 & 108.33 & 2.98 & 2.63 & 12.60 & 12.46 & 81.10 & 81.14 & 19.44 & 18.79 & 1184 & 1136 \\
\hline Giza 843 & 108.33 & 108.33 & 2.08 & 2.02 & 7.97 & 8.09 & 85.29 & 85.72 & 16.84 & 16.05 & 1188 & 1112 \\
\hline Misr 1 & 106.67 & 108.75 & 2.27 & 2.27 & 9.04 & 9.36 & 88.26 & 87.39 & 18.07 & 17.10 & 1152 & 1088 \\
\hline Giza 40 & 95.83 & 96.67 & 2.03 & 1.78 & 8.41 & 8.25 & 76.37 & 76.23 & 16.36 & 15.67 & 1116 & 980 \\
\hline LSD $_{0.05}$ Cultivars & 3.46 & 3.69 & 0.17 & 0.15 & 0.39 & 0.25 & 0.59 & 0.95 & 0.43 & 1.26 & 66.75 & 77.71 \\
\hline \multicolumn{13}{|c|}{ Pesticides treatments } \\
\hline Contr & 103.33 & 106.39 & 2.37 & 2.28 & 8.78 & 9.41 & 76.63 & 76.44 & 15.61 & 14.89 & 1016 & 932 \\
\hline Thiamethoxam & 106.39 & 108.44 & 2.34 & 2.44 & 12.13 & 12.02 & 84.29 & 84.73 & 19 & 59 & 1320 & 1284 \\
\hline Diniconazole & 103.33 & 101.67 & 2.24 & 1.94 & 9.76 & 9.54 & 84.32 & 85.15 & 17.60 & 16.63 & 1168 & 1108 \\
\hline $\begin{array}{l}\text { Thiamethoxam + } \\
\text { diniconazole }\end{array}$ & 106.94 & 105.83 & 2.44 & 2.09 & 8.84 & 9.14 & 82.66 & 82.24 & 15.70 & 15.06 & 1068 & 980 \\
\hline $\begin{array}{l}\mathrm{LSD}_{0.05} \text { Pesticides } \\
\text { treatments. }\end{array}$ & 2.83 & 3.01 & 0.14 & 0.12 & 0.32 & 0.20 & 0.48 & 0.78 & 0.35 & 1.03 & 55.90 & 65.72 \\
\hline
\end{tabular}

Table 2. Effect of interaction between faba bean cultivars and pesticides on plant height (cm), number of branches/plant, number of pods/plant, 100-seed weight $(\mathrm{g})$, seed yield/plant $(\mathrm{g})$ and seed yield/plot $(\mathrm{kg})$ in 2016/17 and 2017/18 growing seasons.

\begin{tabular}{|c|c|c|c|c|c|c|c|c|c|c|c|c|c|}
\hline \multirow{2}{*}{$\begin{array}{l}\text { Traits } \\
\text { Factors }\end{array}$} & & \multicolumn{2}{|c|}{$\begin{array}{l}\text { Plant height } \\
\text { (cm) }\end{array}$} & \multicolumn{2}{|c|}{$\begin{array}{c}\text { No. of branches / } \\
\text { plant }\end{array}$} & \multicolumn{2}{|c|}{$\begin{array}{c}\text { No. of } \\
\text { Pods / plant }\end{array}$} & \multicolumn{2}{|c|}{$\begin{array}{c}\text { 100-seed } \\
\text { weight (g) }\end{array}$} & \multicolumn{2}{|c|}{$\begin{array}{l}\text { Seed yield/ } \\
\text { plant }(\mathrm{g})\end{array}$} & \multicolumn{2}{|c|}{$\begin{array}{l}\text { Seed yield/ } \\
\text { feddan (kg) }\end{array}$} \\
\hline & & 20162017 & $2017 / 2018$ & 20162017 & 20172018 & 20162017 & 20172018 & 20162017 & 20172018 & 20162017 & 20172018 & 20162017 & 20172018 \\
\hline \multirow{5}{*}{ Nubaria 1} & & & & & lltivars X & Pesticid & es treatme & nts & & & & & \\
\hline & Control & 93.33 & 96.67 & 2.40 & 2.07 & 7.96 & 9.67 & 81.66 & 82.13 & 14.13 & 13.79 & 1004 & 972 \\
\hline & Thiamethoxam & 123.33 & 120.67 & 2.20 & 2.40 & 13.53 & 13.20 & 85.03 & 86.3 & 20 & 19.67 & 1460 & 1400 \\
\hline & Diniconazole & 106.67 & 106.67 & 3.40 & 3.27 & 14.83 & 16.76 & 82.01 & 82.89 & 16.53 & 15.34 & 1164 & 1076 \\
\hline & $\begin{array}{l}\text { Thiamethoxam } \\
+ \text { diniconazole }\end{array}$ & 105.00 & 106.67 & 1.60 & 1.33 & 10.31 & 10.70 & 82.03 & 83.31 & 13.63 & 12.85 & 960 & 936 \\
\hline \multirow{4}{*}{ Sakha 1} & Control & 101.67 & 103.33 & 1.80 & 1.73 & 5.31 & 5.43 & 65.77 & 64.77 & 12.03 & 11.6 & 848 & 804 \\
\hline & Thiamethoxam & 98.33 & 100.00 & 2.60 & 2.60 & 13.56 & 13.53 & 88.63 & 91.1 & 17.92 & 16.63 & 1240 & 1236 \\
\hline & Diniconazole & 95.00 & 100.00 & 2.47 & 2.20 & 8.9 & 7.94 & 80.07 & 80.93 & 18.67 & 17.77 & 1196 & 1204 \\
\hline & $\begin{array}{l}\text { Thiamethoxam } \\
+ \text { diniconazole }\end{array}$ & 106.67 & 111.67 & 2.47 & 2.13 & 10.58 & 10.75 & 78.11 & 78.08 & 14.28 & 13 & 1008 & 960 \\
\hline \multirow{4}{*}{ Giza 716} & Control & 105.00 & 111.67 & 2.00 & 1.87 & 8.33 & 8.42 & 75.07 & 75.02 & 12.16 & 11.75 & 852 & 824 \\
\hline & Thiamethoxam & 108.33 & 108.33 & 3.20 & 3.27 & 16.09 & 15.49 & 79.14 & 77.85 & 22.23 & 21.67 & 1260 & 1456 \\
\hline & Diniconazole & 120.00 & 108.33 & 2.47 & 1.67 & 14.27 & 13.67 & 85.52 & 86.62 & 21.67 & 20.67 & 1348 & 1272 \\
\hline & $\begin{array}{l}\text { Thiamethoxam } \\
+ \text { diniconazole }\end{array}$ & 113.33 & 105.00 & 4.27 & 3.73 & 11.71 & 12.24 & 84.68 & 07 & 21.7 & 21.06 & 1284 & 984 \\
\hline \multirow{4}{*}{ Giza 843} & Control & 113.33 & 118.33 & 2.80 & 2.53 & 8.67 & 10.17 & 83.09 & 83.16 & 16.78 & 15.83 & 1188 & 1120 \\
\hline & Thiamethoxam & 98.33 & 103.33 & 2.00 & 2.20 & 8.15 & 8.82 & 84.06 & 84.41 & 16.44 & 15.53 & 1164 & 1048 \\
\hline & Diniconazole & 105.00 & 105.00 & 1.80 & 1.67 & 6.62 & 5.78 & 88.86 & 89.87 & 15.4 & 14.64 & 1076 & 988 \\
\hline & $\begin{array}{l}\text { Thiamethoxam } \\
+ \text { diniconazole }\end{array}$ & 116.67 & 106.67 & 1.73 & 1.67 & 8.46 & 7.59 & 85.17 & 85.43 & 18.73 & 18.2 & 1328 & 1292 \\
\hline \multirow{4}{*}{ Misr 1} & Control & 110.00 & 116.67 & 2.80 & 3.00 & 11.65 & 11.12 & 78.8 & 77.99 & 20.37 & 19.02 & 1064 & 1016 \\
\hline & Thiamethoxam & 103.33 & 105.00 & 2.33 & 2.53 & 11.68 & 12.31 & 92.18 & 91.9 & 19.63 & 18.46 & 1392 & 1300 \\
\hline & Diniconazole & 105.00 & 103.33 & 2.33 & 2.13 & 7.25 & 7.07 & 92.08 & 93.26 & 20.27 & 19.34 & 1308 & 1236 \\
\hline & $\begin{array}{l}\text { Thiamethoxam } \\
+ \text { diniconazole }\end{array}$ & 108.33 & 110.00 & 1.60 & 1.40 & 5.58 & 6.93 & 89.97 & 86.41 & 12.02 & 11.59 & 852 & 800 \\
\hline \multirow{4}{*}{ Giza 40} & Control & 96.67 & 91.67 & 2.40 & 2.47 & 10.79 & 11.62 & 75.39 & 75.6 & 18.17 & 17.37 & 1152 & 852 \\
\hline & Thiamethoxam & 106.67 & 113.33 & 1.73 & 1.67 & 9.76 & 8.75 & 76.71 & 76.83 & 20.33 & 19.6 & 1408 & 1272 \\
\hline & Diniconazole & 88.33 & 86.67 & 1.00 & 0.73 & 6.68 & 6.00 & 77.35 & 77.33 & 13.07 & 12.05 & 924 & 880 \\
\hline & $\begin{array}{l}\text { Thiamethoxam } \\
+ \text { diniconazole }\end{array}$ & 91.67 & 95.00 & 3.00 & 2.27 & 6.42 & 6.63 & 76.02 & 75.15 & 13.85 & 13.67 & 980 & 916 \\
\hline \multicolumn{2}{|c|}{ LSD $_{0.05}$ CVS. X Pest. Treat. } & 6.92 & 7.38 & 0.45 & 0.31 & 0.79 & 0.49 & 1.17 & 1.91 & 0.86 & 2.51 & 93.78 & 99.84 \\
\hline
\end{tabular}


Table 3. Means of Chlorophyll a, Chlorophyll b, Total carbohydrate percentage, Total protein percentage, Peroxidase activity and polyphenoloxid activity as affected by faba bean cultivars and pesticides in 2016/17 and 2017/18 growing seasons.

\begin{tabular}{|c|c|c|c|c|c|c|c|c|c|c|c|c|}
\hline \multirow{2}{*}{$\begin{array}{l}\text { Traits } \\
\text { Factors }\end{array}$} & \multicolumn{2}{|c|}{$\begin{array}{c}\text { Chlorophyll } \\
\text { a }\end{array}$} & \multicolumn{2}{|c|}{$\begin{array}{c}\text { Chlorophyll } \\
\text { b }\end{array}$} & \multicolumn{2}{|c|}{$\begin{array}{c}\text { Total } \\
\text { carbohydrate }(\%)\end{array}$} & \multicolumn{2}{|c|}{$\begin{array}{l}\text { Total protein } \\
\text { (\%) }\end{array}$} & \multicolumn{2}{|c|}{$\begin{array}{l}\text { Peroxidase } \\
\text { activity }\end{array}$} & \multicolumn{2}{|c|}{$\begin{array}{c}\text { polyphenoloxid } \\
\text { activity }\end{array}$} \\
\hline & 2016/17 & $2017 / 18$ & 2016/17 & $2017 / 18$ & 2016/17 & 2017/18 & 2016/17 & $2017 / 18$ & 2016/17 & $2017 / 18$ & 2016/17 & $2017 / 18$ \\
\hline \multicolumn{13}{|c|}{ tiva } \\
\hline Nubaria 1 & 0.52 & 0.54 & 0.37 & 0.38 & 45.98 & 47.36 & 22.41 & 23.08 & 0.45 & 0.46 & 0.42 & 0.44 \\
\hline Sakha & 0.74 & 0.76 & 0.53 & 0.54 & 49.05 & 50.52 & 21.91 & 22.57 & 0.52 & 0.53 & 9 & 0.51 \\
\hline Giza 716 & 0.69 & 0.71 & 0.49 & 0.51 & 48.63 & 50.09 & 22.51 & 23.19 & 0.48 & 0.50 & 0.46 & 0.47 \\
\hline Giza 843 & 0.78 & 0.81 & 0.56 & 0.57 & 51.67 & 53.22 & 22.91 & 23.59 & 0.55 & 0.57 & 0.52 & 0.54 \\
\hline Misr 1 & 0.59 & 0.59 & 0.42 & 0.42 & 49.80 & 50.50 & 22.69 & 23.03 & 0.41 & 0.42 & 0.39 & 0.40 \\
\hline Giza 40 & 0.80 & 0.82 & 0.56 & 0.58 & 51.75 & 53.12 & 24.21 & 24.85 & 0.54 & 0.56 & 0.52 & 0.53 \\
\hline LSD $_{0.05}$ Cultivars & 0.050 & 0.060 & 0.040 & 0.410 & 2.530 & 2.620 & 0.940 & 0.890 & 0.320 & 0.030 & 0.030 & 0.033 \\
\hline \multicolumn{13}{|c|}{ Pesticides treatments } \\
\hline Control & 0.61 & 0 . & 0.43 & 0.44 & 50.87 & 52.36 & 23.55 & 24 & 0.6 & 0.70 & 0.64 & 0.66 \\
\hline Thiamethoxam & 0.67 & 0.69 & 0.45 & 0.46 & 49.62 & 51.07 & 22.59 & 23.24 & 0.55 & 0.57 & 0.52 & 0.54 \\
\hline Diniconazole & 0.78 & 0.80 & 0.51 & 0.52 & 45.86 & 47.19 & 22.06 & 22.71 & 0.43 & 0.44 & 0.41 & 0.42 \\
\hline $\begin{array}{l}\text { Thiamethoxam + } \\
\text { diniconazole }\end{array}$ & 0.69 & 0.70 & 0.57 & 0.58 & 51.71 & 52.73 & 23.02 & 23.49 & 0.30 & 0.31 & 0.29 & 0.29 \\
\hline $\begin{array}{l}\mathrm{LSD}_{0.05} \text { Pesticides } \\
\text { treatments. }\end{array}$ & 0.021 & 0.019 & 0.017 & 0.016 & 0.680 & 0.510 & 0.360 & 0.300 & 0.009 & 0.009 & 0.010 & 0.009 \\
\hline
\end{tabular}

3-Cultivars $x$ pesticides effects:

The results presented in Table (4) indicated that the six cultivars had different responses to the four treatments.

The results showed that the interactions between Giza 40 and Giza 843 with thiamethoxam, diniconazole and Giza 843 with thiamethoxam + diniconazole had excellent mean values for pigments content (chlorophyll $a$ and $b$ ) in both seasons. The interactions between Giza $40 \mathrm{x}$ diniconazole, Giza 716 x thiamethoxam + diniconazole obtained the highest total carbohydrate percentage among all interactions in both seasons. For total protein percentage, Giza 843 and Giza 40 x thiamethoxam + diniconazole showed the protein percentage but these values did not exceeded the values of Giza 716 and Misr 1 in the control treatment. All cultivars under control treatment and the two cultivars Giza 843 and Giza 40 under the thiamethoxam treatment showed highly peroxidase and poly phenol oxidase compared to all other treatments in both seasons.

Table 4. Effect of interaction between faba bean cultivars and pesticides on Chlorophyll a, Chlorophyll b, Total carbohydrate percentage, Total protein percentage, Peroxidase activity and polyphenoloxid activity in 2016/17 and 2017/18 growing seasons.

\begin{tabular}{|c|c|c|c|c|c|c|c|c|c|c|c|c|c|}
\hline \multirow{2}{*}{$\frac{\text { Traits }}{\text { Factors }}$} & & \multicolumn{2}{|c|}{$\begin{array}{c}\text { Chlorophyll } \\
\text { a } \\
\end{array}$} & \multicolumn{2}{|c|}{$\begin{array}{c}\text { Chlorophyll } \\
\text { b }\end{array}$} & \multicolumn{2}{|c|}{$\begin{array}{c}\text { Total } \\
\text { carbohydrate }(\%) \\
\end{array}$} & \multicolumn{2}{|c|}{$\begin{array}{c}\text { Total protein } \\
(\%)\end{array}$} & \multicolumn{2}{|c|}{$\begin{array}{c}\text { Peroxidase } \\
\text { activity }\end{array}$} & \multicolumn{2}{|c|}{$\begin{array}{c}\text { polyphenoloxid } \\
\text { activity }\end{array}$} \\
\hline & & 20162017 & 20172018 & 20162017 & $2017 / 2018$ & 20162017 & $2017 / 2018$ & 20162017 & 20172018 & 20162017 & 20172018 & 20162017 & 20172018 \\
\hline \multirow{5}{*}{ Nubaria } & & & & & ultivars $Y$ & X Pestici & es treatm & ents & & & & & \\
\hline & Control & 0.43 & 0.45 & 0.31 & 0.32 & 50.19 & 51.69 & 22.37 & 23.04 & 0.65 & 0.67 & 0.62 & 0.64 \\
\hline & Thiamethoxam & 0.48 & 0.50 & 0.32 & 0.33 & 42.60 & 43.88 & 23.59 & 24.30 & 0.55 & 0.57 & 0.52 & 0.54 \\
\hline & Diniconazole & 0.65 & 0.67 & 0.43 & 0.44 & 44.49 & 45.82 & 23.93 & 24.64 & 0.42 & 0.43 & 0.40 & 0.41 \\
\hline & $\begin{array}{l}\text { Thiamethoxam } \\
\text { + diniconazole }\end{array}$ & 0.52 & 0.53 & 0.42 & 0.44 & 48.62 & 50.08 & 21.37 & 22.01 & 0.23 & 0.23 & 0.22 & 0.22 \\
\hline \multirow{4}{*}{ Sakha 1} & Control & 0.65 & $\overline{06}$ & 0.46 & 0.47 & 49.99 & 1.49 & 22.96 & 23.65 & 0.72 & 0.74 & 0.68 & 0.70 \\
\hline & Thiamethoxam & 0.72 & 0.14 & 0.48 & 0.49 & 52.29 & 53.85 & 21.14 & 21.77 & 0.56 & 0.58 & 0.53 & 0.55 \\
\hline & Diniconazole & 0.82 & 0.84 & 0.54 & 0.55 & 41.62 & 42.87 & 20.34 & 20.95 & 0.46 & 0.47 & 0.43 & 0.45 \\
\hline & $\begin{array}{l}\text { Thiamethoxam } \\
\text { + diniconazole }\end{array}$ & 0.77 & 0.79 & 0.63 & 0.65 & 52.31 & 53.88 & 23.21 & 23.91 & 0.34 & 0.35 & 0.32 & 0.33 \\
\hline \multirow{4}{*}{ Giza 716} & & 0 & 0.62 & 0.43 & 0.44 & 67 & 07 & 25 & 25.92 & 0.67 & 0. & 0.64 & 0.66 \\
\hline & Thian & 0.67 & & 0 & 0.46 & & & & & 0.52 & & 0.50 & 0.51 \\
\hline & Diniconazole & 0.76 & 0.79 & 0.50 & 0.52 & 40.25 & 41.46 & 21.83 & 22.48 & 0.43 & 0.44 & 0.40 & 0.42 \\
\hline & $\begin{array}{l}\text { Thiamethoxam } \\
\text { + diniconazole }\end{array}$ & 0.72 & 0.74 & 0.59 & 0.61 & 54.26 & 55.89 & 22 & 23.22 & 0.32 & 0.33 & 0.30 & 0.31 \\
\hline \multirow{4}{*}{ Giza 84} & & 0.69 & & 0.48 & 0.50 & 76 & 37 & 22 & 23. & 0.76 & 0 . & 0.72 & 0.74 \\
\hline & Thiar & 0.76 & 0 & 0 & 0.52 & 51.06 & 52.60 & 24 & & 0.59 & 0. & 0.56 & 0.58 \\
\hline & Diniconazole & 0.87 & 0.89 & 0.57 & 0.59 & 49.59 & 51.07 & 20.61 & 21.23 & 0.48 & 0.50 & 0.46 & 0.47 \\
\hline & $\begin{array}{l}\text { Thiamethoxam } \\
\text { + diniconazole }\end{array}$ & 0.82 & 0.84 & 0.67 & 0.69 & 52.27 & 53.84 & 24.56 & 25.29 & 0.36 & 0.37 & 0.34 & 0.35 \\
\hline \multirow{4}{*}{ Misr 1} & Control & 0.51 & 0.52 & 0.36 & 77 & 98 & 55.60 & 24 & 25.36 & 0.56 & 0. & 0.53 & 0.55 \\
\hline & Thiam & 0.56 & 0.58 & 0 & 0.39 & 49.74 & 51.23 & 22.03 & 22 & 0.44 & 0.45 & 0.42 & 0.43 \\
\hline & Diniconazole & 0.64 & 0.66 & 0.42 & 0.43 & 45.78 & 47.15 & 22.84 & 23.53 & 0.36 & 0.37 & 0.34 & 0.35 \\
\hline & $\begin{array}{l}\text { Thiamethoxam } \\
\text { + diniconazole }\end{array}$ & 0.63 & 0.61 & 0.52 & 0.50 & 49.71 & 48.01 & 21.27 & 20.55 & 0.28 & 0.27 & 0.26 & 0.25 \\
\hline \multirow{4}{*}{ Giza 40} & & 0.74 & 0.76 & 0.52 & 0.54 & 50.6 & 51.92 & 23. & 24 & 0.7 & 0 . & 0.67 & 0.68 \\
\hline & Thian & 0.83 & 0.8 & 0.55 & 0.56 & 48.69 & 49.93 & 24.23 & 24.84 & 0.64 & 0.66 & 0.61 & 0.63 \\
\hline & Diniconazole & 0.94 & 0.96 & 0.62 & 0.63 & 54.61 & 55.99 & 23.66 & 24.26 & 0.52 & 0.54 & 0.50 & 0.51 \\
\hline & $\begin{array}{l}\text { Thiamethoxam } \\
\text { + diniconazole }\end{array}$ & 0.69 & 0.71 & 0.56 & 0.58 & 53.05 & 54.65 & 25.20 & 25.95 & 0.30 & 0.31 & 0.29 & 0.30 \\
\hline $\begin{array}{l}\text { LSD } 0.05 \\
\text { Treat. }\end{array}$ & ${ }_{5}$ CVS. X Pest. & 41 & 0.039 & 0.034 & 0.033 & 1.347 & 1.001 & 0.705 & 0.595 & 0.019 & 0.019 & 0.019 & 0.018 \\
\hline
\end{tabular}


3- Effect of cultivars, pesticides and their interaction on white fly and aphid number in 2016/17 and 2017/18 growing seasons:

\section{1- Cultivars effect:}

The presented results in Table (5) indicated that, the lowest numbers of leaf miner (Liromiza congesta) in both seasons were counted in Misr $1 \mathrm{cv}$. (6.44 and 7.38) followed by Nubaria 1 (7.12 and 8.15) then Giza 40 (7.64 and 8.88). While, the largest numbers of leaf miner (Liromiza congesta) were collected from Sakha $1 \mathrm{cv}$. (12.00 and 11.70) in both seasons respectively. In the same way, the lowest numbers of aphid were collected from Giza 40 (6.48 and 8.18) followed by Misr 1 (6.87 and 8.15) then Nubaria 1 (7.48 and 8.86) in the two seasons respectively. On the other hand, the largest numbers of aphid were counted in Giza $716 \mathrm{cv}$. $(9.70$ and 8.75$)$ in $1^{\text {st }}$ and $2^{\text {nd }}$ seasons respectively. These results are in contrast with those by Salman et al. (2006), who found that Giza 40 was low resistance to aphid infestation. Also, Soffan (2012) reported that Gazira 2 faba bean cultivar was less preferred by the cowpea aphid as compared with Misr 1 cultivar.

\section{2-Pesticides effect:}

The obtained results in Table (5) showed that, faba bean plants under the thiamethoxam gave the lowest mean values for numbers of leaf miner (Liromiza congesta) numbers (2.89 and 3.06) and aphid (3.89 and 4.33) in both seasons respectively followed by treated faba bean with thiamethoxam + diniconazole with averages of 8.97 and 9.06 for leaf miner (Liromiza congesta) and 7.49 and 7.89 for aphid in $1^{\text {st }}$ and $2^{\text {nd }}$ seasons respectively. On the other side, the highest numbers of leaf miner (Liromiza congesta) and aphid were obtained under control treatment in both seasons with averages of 12.94 and 13.22 for leaf miner (Liromiza congesta) and 10.61 and 11.28 for aphid in $1^{\text {st }}$ and $2^{\text {nd }}$ seasons respectively.

Our result indicated that Thiamethoxam had a large effect in reducing leaf miner (Liromiza congesta) and aphid numbers in faba bean and these finding in agree with those by Sharma and Lal (2002) which reported that Thiamethoxam insecticide was superior insecticide against leaf miner. after one day of first spray of thiamethoxam (25 $\mathrm{g}$ a.i./ha) the leaf miner population was reduced by 94.80 , $93.74,88.38,86.25$ and 80.70 per cent, respectively on plant. Sreekanth and Reddy (2011) revealed that the most effective insecticides for aphids and whitefly up to seven days was thiomethoxam. Abbas et al. (2012) evaluated three insecticides, including Actara (thiomethoxam) 24\% WG on leaf miner and he found that Actara proved to be highly effective against whitefly. El-Naggar and Zidan (2013) evaluated the effectiveness of thiamethoxam, used separately as foliar applications at the recommended rate against the sucking insects whitefly, Bemicia tabaci, and cotton aphid, Aphis gossypii (Glover) during the 2010 and 2011 in cotton growing seasons. Treatments with thiamethoxam were highly effective against aphids, up to 14 days, while the effect was moderate on the whitefly population (mature and immature stages).

\section{3- Cultivars $x$ pesticides effects:}

The results presented in Table (6) indicated that the six cultivars had different responses to the four treatments. The results showed that spraying the thiamethoxam led to sharply decrease in numbers of leaf miner (Liromiza congesta) and aphid in cultivars under study compared to control. Only the three cultivars Nubaria 1, Misr 1 and Giza 40 showed a large decrease in numbers of leaf miner (Liromiza congesta) and aphid under thiamethoxam + diniconazole treatment. Our result in the same way with those by: Patnaik et al. (2010) evaluated the efficacy of thiamethoxam, diafenthiuron and clothianidin against whitefly Dialeuropora decempuncta during August to November 2009 in West Bengal, India. Amongst the 3 insecticides tested, thiamethoxam at $0.015 \%$ reduced the population of whitefly and leaf miner on mulberry by $99.81 \%$ followed by diafenthiuron at $0.0633 \%$ by $99.62 \%$ and clothianidin at $0.0047 \%$ by $99.07 \%$ on 1 day of spray and $99.81 \%, 99.19 \%$ and 99.07 on 3day of spray, respectively. El- Sayad (2013) evaluated the efficacy of four insecticides for the control of $B$. tabaci on tomato plants. Results showed significant differences on the mean number of $B$. tabaci recorded from different treatments under field conditions. Thiacloprid gave highly reduction in the mean number of $B$. tabaci (1.33 nymph/leaf) than thiamethoxam(1.82 nymph/leaf). Imidacloprid gave a good reduction in the mean number of $B$. tabaci (0.97). Saner et al. (2013) found that field studies Population of leaf miner was effectively suppressed by thiamethoxam $25 \%$ WG, acetamiprid $20 \%$ SP, fipronil $80 \mathrm{WG}$ followed by fipronil 5\% SC, imidacloprid 70\% WG, imidacloprid $17.80 \% \mathrm{SL}$, lambdacyhalothrin $5 \% \quad \mathrm{SC}$ and triazophos $40 \%$ EC. Omprakash and Raju (2013) indicated that neonicotinoids (imidacloprid and thiamethoxam) showed higher efficacy against field populations of $B$. tabaci in reducing pest population. Combination treatments like azadirachtin + thiamethoxam, azadirachtin+ Imidacloprid, azadirachtin + triazophos and azadi rachtin + spinosad showed moderate efficacy and azadirachtin at $1500 \mathrm{ppm}(43.26 \%)$ was the least effective.

4- Effect of cultivars, pesticides and their interaction on disease severity in 2016/17 and 2017/18 growing seasons:

1- Cultivars effect:

The results presented in Table (5) showed that, Nubaria $1 \mathrm{cv}$. was the most resistant one in this study to chocolate spot where it scored the lowest disease severity among all cultivars in both seasons with percentages of $3.70 \%$ and $3.82 \%$ followed by Misr $1 \mathrm{cv}$. with percentages of $4.35 \%$ and $4.44 \%$ in $1^{\text {st }}$ and $2^{\text {nd }}$ seasons respectively. On the other side, Giza $40 \mathrm{cv}$. was the most sensitive cultivars among all tested cultivars it scored the highest disease severity in both seasons $(6.16 \%$ and $6.31 \%)$ followed by Giza 716 (5.85\% and 6.03\%)

\section{2- Pesticides effect:}

The obtained results in Table (5) indicated that the lowest chocolate spot disease severity was recorded from thiamethoxam + diniconazole $(2.59 \%$ and $2.65 \%)$ followed by diniconazole treatment $(3.63 \%$ and $2.65 \%)$ then thiamethoxam $(6.56 \%$ and $6.75 \%)$ in the $1^{\text {st }}$ and $2^{\text {nd }}$ seasons respectively. On the other side the highest chocolate spot disease severity was recorded from control treatment with percentages of $7.72 \%$ and $7.95 \%$ in both seasons respectively. 


\section{3- Cultivars x pesticides effects:}

The presented results in Table (6) indicated that the six cultivars had different responses to the four treatments. The results showed that spraying faba bean plant with the diniconazole and thiamethoxam + diniconazole led to sharply decrease in disease severity in cultivars under study compared to control and thiamethoxam treatment.

Table 5. Means of leaf miner numbers, Aphid numbers and chocolate spot disease severity percentage as affected by faba bean cultivars and pesticides in $2016 / 17$ and 2017/18 growing seasons.

\begin{tabular}{|c|c|c|c|c|c|c|}
\hline \multirow{2}{*}{$\begin{array}{l}\text { Traits } \\
\text { Factors }\end{array}$} & \multicolumn{2}{|c|}{$\begin{array}{c}\text { leaf miner (Liromiza congesta) } \\
\text { numbers }\end{array}$} & \multicolumn{2}{|c|}{$\begin{array}{c}\text { Aphid (Aphis gossypii) } \\
\text { numbers }\end{array}$} & \multicolumn{2}{|c|}{$\begin{array}{c}\text { Chocolate spot } \\
\text { disease severity \% }\end{array}$} \\
\hline & 2016/17 & $2017 / 18$ & 2016/17 & $2017 / 18$ & 2016/17 & $2017 / 18$ \\
\hline \multicolumn{7}{|c|}{ Cultivars } \\
\hline Nubaria 1 & 7.12 & 8.15 & 7.48 & 8.65 & 3.70 & 3.82 \\
\hline Sakha 1 & 12.00 & 11.70 & 8.37 & 7.80 & 5.53 & 5.70 \\
\hline Giza 716 & 11.53 & 9.85 & 9.70 & 8.75 & 5.17 & 5.32 \\
\hline Giza 843 & 9.95 & 9.90 & 8.42 & 8.93 & 5.85 & 6.03 \\
\hline Misr 1 & 6.44 & 7.38 & 6.87 & 8.15 & 4.35 & 4.44 \\
\hline Giza 40 & 7.64 & 8.88 & 6.48 & 8.18 & 6.16 & 6.31 \\
\hline LSD $_{0.05}$ Cultivars & 0.88 & 1.13 & 0.95 & N.S. & 0.32 & 0.34 \\
\hline \multicolumn{7}{|c|}{ Pesticides treatments } \\
\hline Control & 12.94 & 13.22 & 10.61 & 11.2 & 7.72 & 7.95 \\
\hline Thiamethoxam & 2.89 & 3.06 & 3.89 & 4.33 & 6.56 & 6.75 \\
\hline Diniconazole & 11.65 & 11.90 & 9.55 & 10.15 & 3.63 & 3.73 \\
\hline Thiamethoxam + diniconazole & 8.97 & 9.06 & 7.49 & 7.89 & 2.59 & 2.65 \\
\hline LSD $_{0.05}$ Pesticides treatments. & 0.52 & 0.52 & 0.60 & 0.60 & 0.09 & 0.08 \\
\hline
\end{tabular}

N.S. indicates not significant differences according to LSD.

Table 6. Effect of interaction between faba bean cultivars and pesticides on leaf miner numbers, Aphid numbers and chocolate spot disease severity percentage in 2016/17 and 2017/18 growing seasons.

\begin{tabular}{|c|c|c|c|c|c|c|c|}
\hline \multirow{2}{*}{$\frac{\text { Traits }}{\text { Factors }}$} & & \multicolumn{2}{|c|}{$\begin{array}{c}\text { leaf miner (Liromiza congesta) } \\
\text { numbers }\end{array}$} & \multicolumn{2}{|c|}{$\begin{array}{c}\text { Aphid (Aphis gossypii) } \\
\text { numbers }\end{array}$} & \multicolumn{2}{|c|}{$\begin{array}{c}\text { Chocolate spot } \\
\text { disease severity \% }\end{array}$} \\
\hline & & $2016 / 17$ & $2017 / 18$ & $2016 / 17$ & $2017 / 18$ & $2016 / 17$ & $2017 / 18$ \\
\hline \multicolumn{8}{|c|}{ Cultivars X Pesticides treatments } \\
\hline \multirow{4}{*}{ Nubaria 1} & Control & 9.67 & 11.00 & 9.33 & 11.00 & 5.55 & 5.72 \\
\hline & Thiamethoxam & 3.33 & 4.00 & 5.67 & 6.00 & 4.72 & 4.86 \\
\hline & Diniconazole & 8.70 & 9.90 & 8.40 & 9.90 & 2.61 & 2.69 \\
\hline & Thiamethoxam + diniconazole & 6.77 & 7.70 & 6.53 & 7.70 & 1.94 & 2.00 \\
\hline \multirow{4}{*}{ Sakha 1} & Control & 16.67 & 16.33 & 11.33 & 10.33 & 8.29 & 8.54 \\
\hline & Thiamethoxam & 4.67 & 4.33 & 4.00 & 4.33 & 7.05 & 7.26 \\
\hline & Diniconazole & 15.00 & 14.70 & 10.20 & 9.30 & 3.90 & 4.01 \\
\hline & Thiamethoxam + diniconazole & 11.67 & 11.43 & 7.93 & 7.23 & 2.90 & 2.99 \\
\hline \multirow{4}{*}{ Giza 716} & Control & 16.33 & 14.00 & 13.00 & 11.67 & 7.74 & 7.97 \\
\hline & Thiamethoxam & 3.67 & 3.00 & 5.00 & 4.67 & 6.58 & 6.78 \\
\hline & Diniconazole & 14.70 & 12.60 & 11.70 & 10.50 & 3.64 & 3.75 \\
\hline & Thiamethoxam + diniconazole & 11.43 & 9.80 & 9.10 & 8.17 & 2.71 & 2.79 \\
\hline \multirow{4}{*}{ Giza 843} & Control & 14.67 & 14.33 & 11.67 & 12.33 & 8.77 & 9.03 \\
\hline & Thiamethoxam & 1.67 & 2.33 & 3.33 & 3.67 & 7.45 & 7.68 \\
\hline & Diniconazole & 13.20 & 12.90 & 10.50 & 11.10 & 4.12 & 4.25 \\
\hline & Thiamethoxam + diniconazole & 10.27 & 10.03 & 8.17 & 8.63 & 3.07 & 3.16 \\
\hline \multirow{4}{*}{ Misr 1} & Control & 9.33 & 10.67 & 9.67 & 11.33 & 6.48 & 6.67 \\
\hline & Thiamethoxam & 1.33 & 2.00 & 2.67 & 3.67 & 5.51 & 5.67 \\
\hline & Diniconazole & 8.40 & 9.60 & 8.70 & 10.20 & 3.05 & 3.14 \\
\hline & Thiamethoxam + diniconazole & 6.70 & 7.25 & 6.46 & 7.42 & 2.36 & 2.28 \\
\hline \multirow{4}{*}{ Giza 40} & Control & 11.00 & 13.00 & 8.67 & 11.00 & 9.50 & 9.74 \\
\hline & Thiamethoxam & 2.67 & 2.67 & 2.67 & 3.67 & 8.08 & 8.28 \\
\hline & Diniconazole & 9.90 & 11.70 & 7.80 & 9.90 & 4.47 & 4.58 \\
\hline & Thiamethoxam + diniconazole & 7.00 & 8.17 & 6.77 & 8.17 & 2.58 & 2.66 \\
\hline $\mathrm{LSD}_{0.05} \mathrm{Cl}$ & X Pest. Treat. & 1.07 & 1.05 & 1.21 & 1.23 & 0.18 & 0.17 \\
\hline
\end{tabular}

5- The relationship between the activity of defense enzymes (peroxidase and polyphenol oxidase) and chocolate spot disease severity on faba bean cultivars:

Results presented in Figs. 1,2,3 and 4 indicated that that there were highly significant positive associated between disease severity of chocolate spots with peroxidase and polyphenol oxidase in both seasons. The associated between disease severity and peroxidase were significant positive with correlation coefficient of 0.929 and 0.905 while, the associated between disease severity and polyphenol oxidase were highly significant positive with correlation coefficient of 0.930 and 0.897 .

These results are in agree with Kumari and Vengadaramana (2017) who reported that plants enhance defense responses by inducing activity of a broad spectrum 
of defense enzymes which are pathogenesis - related (PR) protiens, namely peroxidase, $\beta-1,3$ - glucanase, chitinase, polyphenol oxidase and phenylalanine ammonia lyase which slow the rate of disease spread. Also, El-Ghanam et al. (2018) mentioned that with increasing the activity of peroxidase and polyphenol oxidase, the disease severity of powdery mildew in squash decreased. Lai et al. (2007) mentioned that, most of the biotic and abiotic stresses lead to an increase in the production of reactive oxygen species

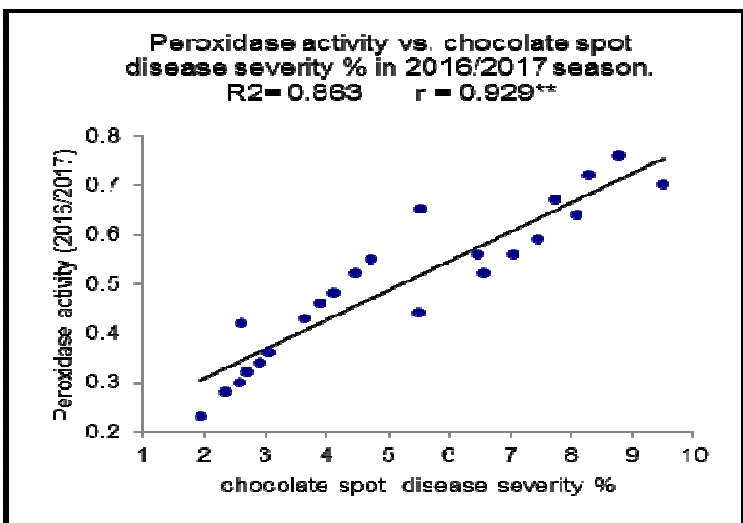

Fig. 1. The relationship between Peroxidase activity and chocolate spot disease severity \% in $2016 / 2017$ season.

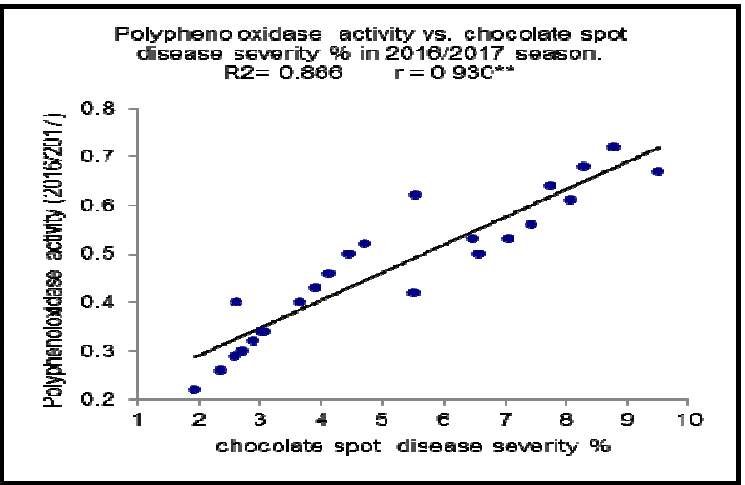

Fig. 3. The relationship between Poly phenoloxidase activity and chocolate spot disease severity \% in 2016/2017 season.

6- Linearity, recovery thiamethoxam, and diniconazole

The pesticides residue had a dangerous effect in human, animals and poultry health. The pesticides with high residue and long half live may due to many dangerous diseases such as cancer. In Egypt most citizens consumed the green faba bean seed and for this reason the measuring of pesticides residue will be very useful to take care of Egyptian citizen health. To ensure quality of the insecticide residue results, the method performance characteristics were generated and evaluated before real broad bean (pods, peels, seeds) samples were analyzed. The recovery percentages thiamethoxam were in the range between 90.21 and $95.73,81.56$ and $90.22,84.81 .1$ and $99.15 \%$, respectively. And diniconazole were in the range between 85.13 and $100.21,80.43$ and $90.11,81.77$ and $90.01 \%$, respectively. These results are considered to be highly satisfactory for the purpose of pesticide residue analysis and they are compliant with the European Union Criteria
(ROS) which in high density, hurt cells lipids, proteins and nucleic acids and finally stop the natural metabolism of plant. Plants protect themselves from cytotoxic effects of these ROS with the help of antioxidant enzymes such as peroxidase, polyphenol moxidase, catalase and superoxide dismutase induced in plants in response to the stress (Joseph and Jini, 2010, He et al., 2011 and Rani and Jyothsna, 2012).

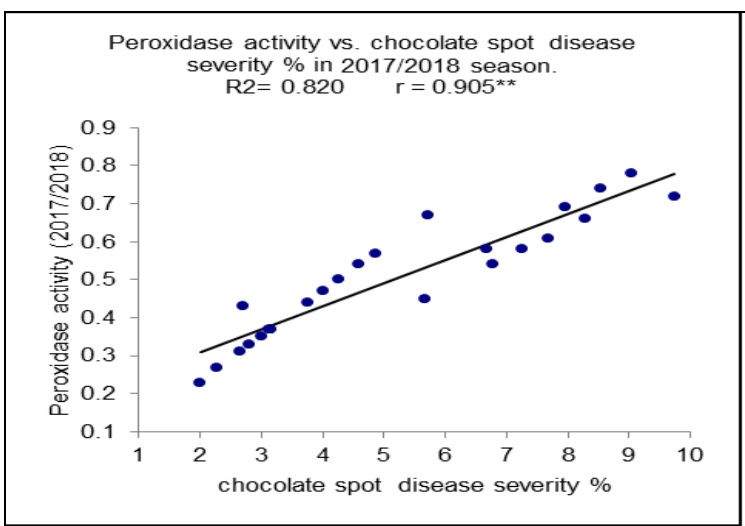

Fig. 2. The relationship between Peroxidase activity and chocolate spot disease severity $\%$ in $2017 / 2018$ season.

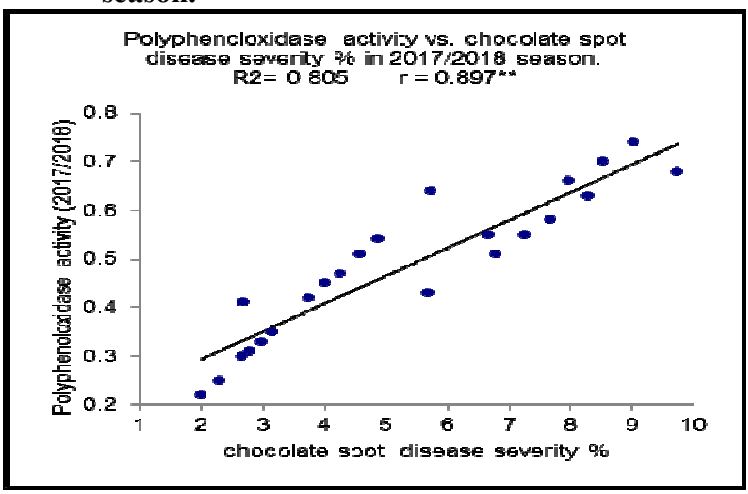

Fig. 4. The relationship between Poly phenoloxidase activity and chocolate spot disease severity \% in 2017/2018 season.

which stipulate the average recoveries in the range 70-120 $\%$ with corresponding RSD less or equal $20 \%$ (SANCO/12495/2011).

\section{Residues of thiamethoxam:}

The residues of thiamethoxam on and in broad bean (pods,peels,seeds). The data showed that the concentration of residues in the initial deposit was $0.621 \mathrm{mg} \mathrm{kg}^{-1}$, tow hour after application. The amount of residues decreased to $0.405 \mathrm{mg} \mathrm{kg}^{-1}$, it gave the rate of loss $34.78 \%$ within the first 24 hours after spray. The residues reduced to 0.311 , $0.175,0.102,0.087,0.035$, and $0.004 \mathrm{mg} \mathrm{kg}^{-1}$ after 3, 7, 10, 15 and 21 days from treatment and the corresponding calculated rates of loss were 49.90, 71.81, 83.57, 85.99, 94.63 , and $99.35 \%$, respectively. The samples taken 21 days after treatment contained no detectable amounts of thiamethoxam in the pods.

In case of peels fruits, the initial deposit of thiamethoxam was $1.802 \mathrm{mg} \mathrm{kg}^{-1}$. This value was dropped to $1.031 \mathrm{mg} \mathrm{kg}^{-1}$, recorded $42.84 \%$ loss, on day after 
application. Residues decreased gradually, at the intervals of $3,5,7,10,15$ and 21 days after treatments, the estimated residues were $0.629,0.472,0.291,0.158,0.931$ and 0.036 $\mathrm{mg} \mathrm{kg}^{-1}$, respectively. Their rates of loss were $65.53,73.80$, $83.85,91.83,94.83$ and $98.002 \%$, respectively. Also, samples of seeds were $00.00,0.058,0.099,0.75,0.43,0.24$ and $0.002 \mathrm{mg} \mathrm{kg}^{-1}$ after0, 1, 3, 5, 7, 10 and 15 days of the treatment respectively.

Detectable amounts of thiamethoxam residues according to the sensitivity of determination procedure. The half-life value of thiamethoxam was $3.02,3.59$, and 2.57 days of the application with degradation rate of 0.138 , 0.051 and 0.269 on for pods, peels and seeds, respectively.

Thiamethoxam is hard to avoid release into the natural environment. Therefore, an evaluation of the safety and efficacy of its applications has become the biggest concern for human health and its maximum residue limits (MRLs) in many crops have been legislated in some countries. In recent years, analytical methods for the determination of thiamethoxam and its dissipation, residue levels at harvest, and other behaviors in soil, water and some crops have been reported (Campbell et al. 2005, Karmakar et al. 2006 and Gupta et al. 2008).

The data revealed that, faba bean could be safely consumed after 24 hours of application according to the recommended maximum residue limit (MRL) for Thiamethoxam in bean $(0.2 \mathrm{ppm})$. These results are in agreement with those obtained by Precheur et al. (1992) and Alaa et al. (2007).

\section{REFERENCES}

Abbas, A. M. J.; M. D. Gogi; S. K. Abbas, and H. Karar (2012). Performance of imidacloprid, Thiomethoxam, acetamaprid and a biocontrol agent (Chrysoperlacarnea) against whitefly, Jassid and thrips on different cotton cultivars. World J. of Zoology; 7(2): 141-146.

Abebe, T.; T. Birhane; Y. Nega and A. Workineh (2014). The Prevalence and Importance of faba Bean diseases with special consideration to the newly emerging "faba bean gall" in Tigary, Ethiopia. Discourse J. of Agric. and Food Sci., 2(2): 33-38.

Abo-Khalil, N.A.; W.A. Al-Murshidy; A.M. Eman and R.A. Badawy (2015). Effect of plant density and calcium nutrition on growth and yield of some faba bean varieties under saline conditions. Journal of International Scientific Publications 3, 440-450.

Abou-Elhagag, G. H. and A. M. A. Salman (2001). Seasonal abundance of certain faba bean pests and their associated predators in Southern Egypt. Assiut J. Agric. Sci., 32: 4 9- 63.

Agegnehu, G. and R. Fessehaie (2006). Response of faba bean to phosphate fertilizer and weed control on nitisols of Ethiopian highlands. Italian J. Agron., 2: 281-290.

Alaa, K.; A. Saleh; I. Samy and A. Mohammed (2007). Degradation of the acaricides abamectin, flufenoxuron and amitraz on Saudi Arabian dates J. Food Chemistry, 100: $1590-1593$.

Ali, M. M. and M. A. Morsy (1983). The importance of natural enemies controlling aphids infesting certain winter crops in Upper Egypt. $5^{\text {th }}$ Arab Pesticide Conf. Tanta Univ. Sept., 2: 332-346.

Amako, A.; K. Chen and K. Asada (1994). Separate assays specific for ascorbiate peroxidase and gauiacol peroxidase and for the chloroplastic and cytosilic isoenxymes of ascorbate peroxidase in plants. Plant Cell Physiol. 35:497-504.
AOAC. (1995). Official Methods of Analysis of International, $16^{\text {th }}$ Edition, Vol. 1, "Agricultural, Chemicals, Contaminants, Drugs" Washington, D. C., USA.

Bakry, B.A.; T.A. Elewa; M.F. El Karamany; M.S. Zeidan and M.M. Tawfik (2011). Effect of row spacing on yield and its components of some faba bean varieties under newly reclaimed sandy soil condition. World Journal Agricultural Science. 7(1), 68-72.

Baliadi, Y. and W. Tengkano (2010). Leafminer, Liriomyza sp. (Diptera: Agromyzidae), a new pest of soybean in Indonesia. Jurnal Penelitian dan Pengembangan Pertanian, 29(1): 1 -9.

Bernier G.; A. Havelange; C. Houssa; A. Petitjean and P. Lejeune (1993). Physiological signals that induce flowering. Plant Cell 5: 1147-1155

Bueno, A.F.; B. Zechmann; W. W. Hoback; R. C. O. F. Bueno and O. A. Fernandes (2007). Serpentine leafminer ( Liriomyza trifolii) on potato (Solanum tubero sum ): field observations and plant photosynthetic responses to injury. Ciencia Rural; 37(6): 1510- 1517.

Campbell, S.; L. Chen; J. Yu and Q.X. Li (2005). Adsorption and analysis of the insecticides thiamethoxam and indoxacarb in Hawaiian soils. J. Agric. Food Chem. 53:5373-5376.

Cheema, H. K.; G. K. Taggar; R. Singh and B.S. Kooner (2009). Evaluation of insecticides against Bemisia tabaci (Gennadius) Mungo (Linnaeus) Hepper on urdbean, Vigna. J. of Inse. Sci (Ludhiana) 22(4):388392.

Dubois, M.; K.A. Gilles; J.K. Hamilton; P.A. Robers and F. Smith (1956). Colorimetric method for determination of sugars and related substances. Analytical Chemistry 28(3): 350-356.

El -Defrawi, G. M.; A. K. Emam; I. A. Marzouk and L. Rizkalla (2000). Population dynamics and seasonal distribution of Aphis craccivora Koch and associated natural enemies in relation to virus disease incidence in faba bean fields. Egyptian Journal of Agricultural Research. 78(2): 627- 641.

El- Sayad, A. I. (2013). Field evaluation of plant extracts and certain insecticides against Bemesia tabaci (Gennadius) on tomato plants and Myzus persicae (Sulzer) on pepper plants. J. of App. Sci. Res.; 9(3):2372-2377.

El-Ghanam, Abeer. A. M.; M. H. Rahhal; M. A. Al-Saman and Entsar, K. A. Khattab (2018). Alternative safety methods for controlling powdery mildew in squash under field conditions. Asian J. Adv. Agric. Res. 7(1): $1-21$.

El-Kholy, R.M.A. (2014). Chemical and biological control of chocolate spot disease in faba bean under field conditions. Middle East J. of Agriculture Research. 3(2): 368-377.

El-Naggar, J. B. and N. E. A. Zidan (2013). Field evaluation of imidacloprid and thiamethoxam against sucking insects and their side effects on soil fauna. J. of Plant Protection Res.; 53(4):375-387.

FAOSTAT (2017).http://www.fao.org/faostat/en/ \# rankings/ countries by commodity.

Gameel, O. I. (1973). Field evaluation of insecticides for jassid, Empoasca lybica De Berg and whitefly, Bemisia tabaci (Genn.) control on cotton. Bull. Entomo. Soc. Egypt, Econo. Ser., 7: 113-122.

Gomez, K.A. and A.A. Gomez (1984). Statistical procedures for Agriculture Research $2^{\text {nd }}$ Ed., Willey and Sons. Inc. New York. USA.

Gupta S, V.T. Gajbhiye and R.K. Gupta (2008). Soil dissipation and leaching behavior of a neonicotinoid insecticide thiamethoxam. Bull Environ Contam Toxicol 80:431437. 
He, J.; F. Chen; S. Chen; G. Lv and Y. Deng (2011). Chrysanthemum leaf epidermal surface morphology and antioxidant and defense enzyme activity in reponse to aphid infestation. J. Plant Physiol., 168 (7): 687-693.

Hendawey M.H. and A.M.A. Younes (2013). Biochemical evaluation of some faba bean cultivars under rainfed conditions at El-Sheikh Zuwayid. Annals of Agricultural Sciences 58(2): 183-193.

ICARDA (International Center for Agricultural Research in the Dryland Areas) (2008). Impact of improved faba bean technologies in Africa No. 2.

Joseph, B. and D. Jini (2010). Insights into the role of antioxidative enzymes for salt tolerance in plants. Int. J. Bot., 6: 456-464.

Karmakar, R.; S.B. Singh and G. Kulshrestha (2006). Persistence and transformation of thiamethoxam, a neonicotinoid insecticide, in soil of different agroclimatic zones of India. Bull. Environ. Contam. Toxicol., 76:400-406.

Kumari, Y. S. M. A. I. and A. Vengadaramana (2017). Stimulation of defense enzymes in tomato (Solanum lycopersicum L.) and chilli (Capsicum annuum L.) in response to exogenous application of different chemical elicitors. Univ. J. Plant Sci., 5(1): 10-15.

Lai ,Q. X.; Z. Y. Bao; Z. G. Zhu; Q. Q. Qian and B. Z. Mao (2007). Effect of osmotic stress on antioxidant enzymes activities in leaf discs of PsaG 12-IPT modified gerbera, J. Zhejiang Univ. Sci., B. 8 (7): 458-464.

Mayer, A. M and E. Harel (1979). Polyphenol oxidases in plants Phytochem., 18 (2):193-215.

Mitiku, A. and M. Wolde (2015). Effect of Faba Bean (Vicia faba L.) Varieties on Yield Attributes at Sinana and Agarfa Districts of Bale Zone, Southeastern Ethiopia. Jordan Journal of Biological Sciences. 8(4): 281-286.

Moran, R. and D. Porath (1980). Chlorophll determination in intact tissues using $\mathrm{N}, \mathrm{N}$-dimethyl form amide. Plant Physiol. 65: 478-479.

Omprakash, S. and S. V. S. Raju (2013). Bioefficacy of some insecticides against Bemisia tabaci (Genn.) on brinjal.Indian Journal of Entomology, 75(4):310-314.

Patnaik, M.; P. Mitra; N. K. Das; K. Mondal and A. K. Bajpai (2010). Field efficacy of some insecticides against whitefly infesting mulberry, Morus alba L. The J. of Plant Protection Sci., 2(2):95-96.
Precheur, R.J.; M.A. Bennett; R.M. Riedel; K.L. Wiese and J. Dudek (1992). Management of fungicide residues on processing tomatoes Plant Disease, 76: 700-702.

Rani, P. U. and Y. Jyothsna (2012). Biochemical and enzymatic changes in rice plants as a mechanism of defense. Acta Physiol. Plant., 32 (4): 695-701.

Rewal, H. S. and J. S. Jhooty (1985). Differential response of wheat varieties to systemic fungicides applied to Ustilago tritici (Pers.). Rostr. Indian J. Agric. Sci., 55: 548-549.

Salman, A. M. A.; A. S. H. Abdel- Moniem and A. H. Obiadalla (2006). Influence of certain agricultural practices on the cowpea aphid, Aphis craccivora Koch, infesting broad bean crops and relation between the infestation and yield of plants in Upper Egypt. Archives of phytopathology and plant protection, 40 ( 6): 395-405.

Saner, D. V.; G. B. Kabre and Y. A. Shinde (2013). Efficacy of newer insecticides on sucking pests in Bt cotton under Khandesh region of Maharashtra. International J. of Plant Protection; 6(2):405-411.

Sharma, D.R. and O.P. Lal (2002). Bio-efficacy of thiamethoxam in comparison to recommended insecticides against leafhopper and whitefly of brinjal (Solanum melongena L.). J. Ent. Res., 26 (3): 257-262.

Soffan, A. (2012). Feeding behavior and biological performance of Cowpea aphid, Aphis craccivora Koch (Hemiptera: Aphididae) on faba bean, Vicia faba L. cultivars. M.Sc. Thesis. Plant protection Dept., Coll. of food \& Agric. Sci., King Saud Univ. Riyadh, K.S.A.

Sreekanth, P. N. and K. M. S. Reddy (2011). Efficacy of different insecticides against sucking pests of cotton. Environment and Ecology, 29(4A):2035-2039.

Teshome, E. and A. Tagegn (2013). Integrated management of chocolate spot (Botiytis fabae Sard.) of faba bean (Vicia $f a b a$ L.) at highlands of Bale, southeastern, Ethiopia. Res. J. of Agric. and Environ. Manag., 2(1): 11-14.

Torres, A. M.; B. Roman; C. M. Avila; Z. Satovic; D. Rubiales; J. I. Sillero and M. T. Moreno (2004). Faba bean breeding for resistance against biotic stresses: towards application of marker technology. Euphytica, 147: 6780

Zebire, D. A. and K. A. Tadesse (2018). Evaluation of faba bean (Vicia faba L.) varieties for yield and reaction to chocolate spot disease at Chencha, Southern Ethiopia. African Journal of Plant Science. 12(8): 155-163.

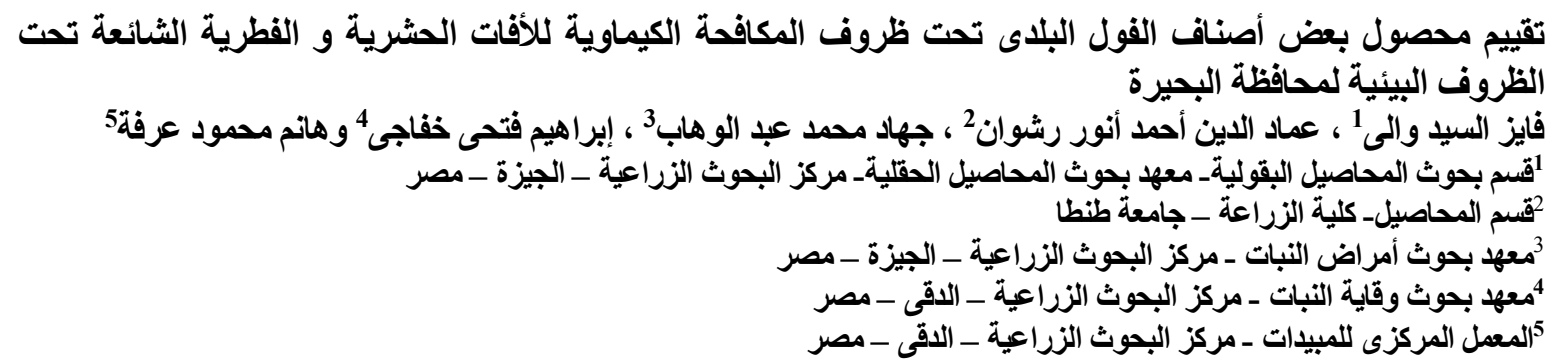

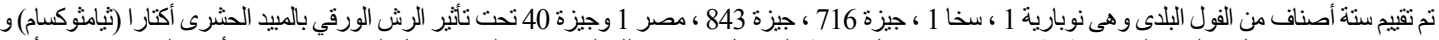

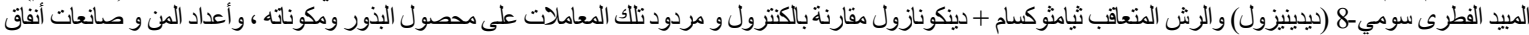

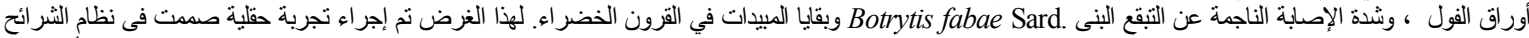

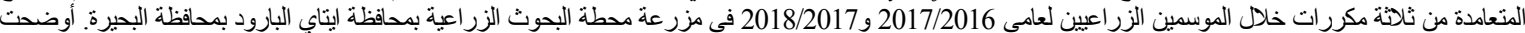

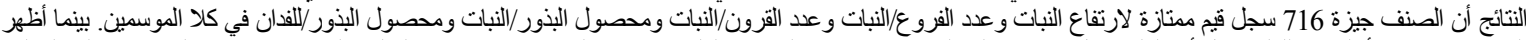

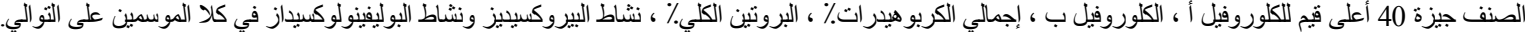

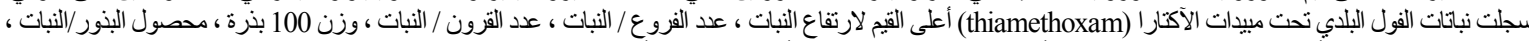

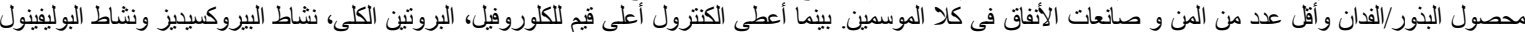

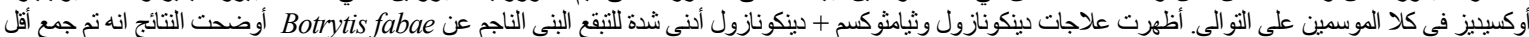

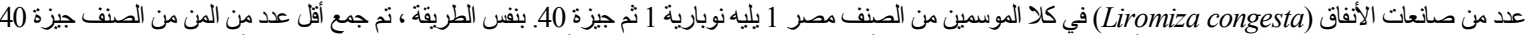

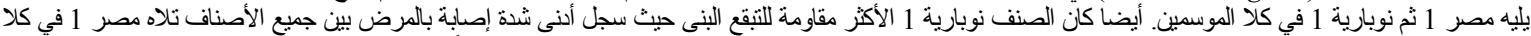

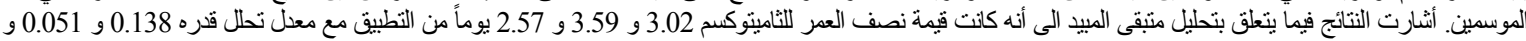
0.269 بالنسبة للقرون و القتشور و البذور على التو الي. و عليه سوف تكون بذور الفول آمنة للاستهلاك البشري بعد 24 ساعة من رش الثيامثنوكسام. 